\title{
EIGENVALUES OF THE LAPLACIAN FOR THE THIRD BOUNDARY VALUE PROBLEM
}

\author{
E. M. E. ZAYED ${ }^{1}$
}

(Received 22 April 1986; revised 12 August 1986 and 28 November 1986)

\begin{abstract}
The spectral function $\theta(t)=\sum_{n-1}^{\infty} \exp ^{\left(-\lambda_{n} t\right)}$, where $\left\{\lambda_{n}\right\}_{n-1}^{\infty}$ are the eigenvalues of the two-dimensional Laplacian, is studied for a variety of domains. The dependence of $\theta(t)$ on the connectivity of a domain and the impedance boundary conditions is analysed. Particular attention is given to a doubly-connected region together with the impedance boundary conditions on its boundaries.
\end{abstract}

\section{Introduction}

The underlying problem is to deduce the precise shape of a membrane from the complete knowledge of the eigenvalues $\lambda_{n}$ for the Laplace operator $\Delta=\partial^{2} / \partial x^{2}$ $+\partial^{2} / \partial y^{2}$ in the $x y$-plane.

Let $D \subseteq R^{2}$ be a bounded domain with a smooth boundary $\partial D$. Consider the impedance problem

$$
(\Delta+\lambda) u=0 \quad \text { in } D, \quad\left(\frac{\partial}{\partial n}+\gamma\right) u=0 \quad \text { on } \partial D,
$$

where $\partial / \partial n$ denotes differentiation along the inward pointing normal to $\partial D, \gamma$ is a positive constant and $u \in C^{2}(D) \cap C(\bar{D})$. Denote its eigenvalues, counted according to multiplicity, by

$$
0<\lambda_{1} \leqslant \lambda_{2} \leqslant \lambda_{3} \leqslant \cdots \leqslant \lambda_{n} \leqslant \cdots \rightarrow \infty \text { as } n \rightarrow \infty .
$$

\footnotetext{
${ }^{1}$ Mathematics Department, Faculty of Science, Zagazig University, Zagazig, Egypt.

(c) Copyright Australian Mathematical Society 1987, Serial-fee code 0334-2700/87
} 
The problem of determining the geometry of $D$ (simply connected) and the impedance $\gamma$ has been discussed recently in [6] from the asymptotic behaviour of the spectral function $\theta(t)=\operatorname{tr}\left(\exp ^{(-\Delta t)}\right)=\sum_{n=1}^{\infty} \exp \left(-\lambda_{n} t\right)$ for small positive $t$. Problem (1.1) has been investigated in [3], [5], [9] in the following special cases:

Case 1. $\gamma=0$ (Neumann Problem)

$$
\theta(t) \sim \frac{\text { area } D}{4 \pi t}+\frac{\text { length } \partial D}{8(\pi t)^{1 / 2}}+a_{0}+\frac{7 t^{1 / 2}}{256 \pi^{1 / 2}} \int_{\partial D}(k(\sigma))^{2} d \sigma+O(t) \text { as } t \rightarrow 0,
$$

Case 2. $\gamma \rightarrow \infty$ (Dirichlet Problem)

$\theta(t) \sim \frac{\text { area } D}{4 \pi t}-\frac{\text { length } \partial D}{8(\pi t)^{1 / 2}}+a_{0}+\frac{t^{1 / 2}}{256 \pi^{1 / 2}} \int_{\partial D}(k(\sigma))^{2} d \sigma+O(t)$ as $t \rightarrow 0$,

where $k(\sigma)$ is the curvature of the boundary $\partial D$. The constant term $a_{0}$ has geometric significance, e.g. if $D$ is smooth and convex, then $a_{0}=1 / 6$ and if $D$ is permitted to have a finite number " $h$ " of smooth convex holes, then $a_{0}=$ $(1-h) / 6$.

The object of this paper is to discuss the following problem: let

$$
D=\{(r, \theta): a \leqslant r \leqslant b, 0 \leqslant \theta \leqslant 2 \pi\}
$$

be a circular annulus. Suppose that the eigenvalues $0<\lambda_{1} \leqslant \lambda_{2} \leqslant \lambda_{3} \leqslant \cdots$ are given for the impedance problem

$$
(\Delta+\lambda) u=0 \quad \text { in } D, \quad\left(\frac{\partial u}{\partial r}+\gamma_{1} u\right)_{r=a}=\left(\frac{\partial u}{\partial r}+\gamma_{2} u\right)_{r=b}=0,
$$

where $\gamma_{1}$ and $\gamma_{2}$ are positive constants. The basic problem is that of determining the geometry of the circular annulus $D$ as well as the impedances $\gamma_{1}$ and $\gamma_{2}$ from the asymptotic behaviour of $\theta(t)$ for small positive $t$.

Problem (1.4) has been investigated in [7] in the following special cases:

Case 1. $\gamma_{1}=\gamma_{2}=0$

$$
\theta(t) \sim \frac{b^{2}-a^{2}}{4 t}+\frac{\pi^{1 / 2}(a+b)}{4 t^{1 / 2}}+O\left(t^{1 / 2}\right) \text { as } t \rightarrow 0 .
$$

Case 2. $\gamma_{1}=0, \gamma_{2} \rightarrow \infty$

$$
\theta(t)-\frac{b^{2}-a^{2}}{4 t}+\frac{\pi^{1 / 2}(a-b)}{4 t^{1 / 2}}+O\left(t^{1 / 2}\right) \text { as } t \rightarrow 0 \text {. }
$$


Case 3. $\gamma_{1} \rightarrow \infty, \gamma_{2}=0$

$$
\theta(t) \sim \frac{b^{2}-a^{2}}{4 t}+\frac{\pi^{1 / 2}(b-a)}{4 t^{1 / 2}}+O\left(t^{1 / 2}\right) \text { as } t \rightarrow 0 .
$$

Case 4. $\gamma_{1}=\gamma_{2} \rightarrow \infty$

$$
\theta(t) \sim \frac{b^{2}-a^{2}}{4 t}-\frac{\pi^{1 / 2}(a+b)}{4 t^{1 / 2}}+O\left(t^{1 / 2}\right) \quad \text { as } t \rightarrow 0 .
$$

A restricted form of the results (1.8) and (1.5) has been obtained recently in $[1,2]$.

With reference to (1.2), (1.3), an examination of the results (1.5)-(1.8) shows that the coefficient of $(4 \pi t)^{-1}$ determines the area of the annulus $D$ and the coefficient of $(\pi t)^{-1 / 2} / 8$ determines the total length of its boundary. We note that the constant term $a_{0}$ is zero because our domain has only one hole (i.e., $h=1)$.

\section{Formulation of the mathematical problem}

Following the method of Kac [3] and following closely the procedure of Section 2 in [7], it is easy to show that the spectral function $\theta(t)$ is given by

$$
\theta(t)=\iint_{D} G(\mathbf{x}, \mathbf{x} ; t) d \mathbf{x},
$$

where $G\left(\mathbf{x}, \mathbf{x}^{\prime} ; t\right)$ is the Green's function for the heat equation

$$
\left(\Delta-\frac{\partial}{\partial t}\right) u=0
$$

subject to the impedance boundary conditions of (1.4) and the initial condition $G\left(\mathbf{x}, \mathbf{x}^{\prime} ; t\right) \rightarrow \delta\left(\mathbf{x}-\mathbf{x}^{\prime}\right)$ as $t \rightarrow 0$, where $\delta\left(\mathbf{x}-\mathbf{x}^{\prime}\right)$ is the Dirac delta function located at the source point $x=x^{\prime}$. Let us write

$$
G\left(\mathbf{x}, \mathbf{x}^{\prime} ; t\right)=G_{0}\left(\mathbf{x}, \mathbf{x}^{\prime} ; t\right)+\chi\left(\mathbf{x}, \mathbf{x}^{\prime} ; t\right),
$$

where

$$
G_{0}\left(\mathbf{x}, \mathbf{x}^{\prime} ; t\right)=(4 \pi t)^{-1} \exp \left\{-\left|\mathbf{x}-\mathbf{x}^{\prime}\right|^{2} / 4 t\right\}
$$

is the "fundamental solution" of the heat equation (2.2), while $\chi\left(\mathbf{x}, \mathbf{x}^{\prime} ; t\right)$ is a "regular solution" chosen in such a way that $G\left(\mathbf{x}, \mathbf{x}^{\prime} ; t\right)$ satisfies the impedance boundary conditions of (1.4). 
On setting $\mathbf{x}=\mathbf{x}^{\prime}$ we find that

$$
\theta(t)=\frac{b^{2}-a^{2}}{4 t}+K(t)
$$

where

$$
K(t)=\iint_{D} \chi(\mathbf{x}, \mathbf{x} ; t) d \mathbf{x} .
$$

The problem now is to determine the asymptotic expansion of $K(t)$ for small positive $t$. In what follows we shall use Laplace transforms with respect to " $t$ ", and use $s^{2}$ as the Laplace transform parameter; thus

$$
\bar{G}\left(\mathbf{x}, \mathbf{x}^{\prime} ; s^{2}\right)=\int_{0}^{\infty} e^{-s^{2} t} G\left(\mathbf{x}, \mathbf{x}^{\prime} ; t\right) d t .
$$

An application of the Laplace transform to the heat equation (2.2) shows that $\bar{G}\left(\mathbf{x}, \mathbf{x}^{\prime} ; s^{2}\right)$ satisfies the membrane equation

$$
\left(\Delta-s^{2}\right) \bar{G}\left(\mathbf{x}, \mathbf{x}^{\prime} ; s^{2}\right)=-\delta\left(\mathbf{x}-\mathbf{x}^{\prime}\right) \text { in } D,
$$

together with the impedance boundary conditions of (1.4). The asymptotic expansion of $K(t)$ for $t \rightarrow 0$ may then be deduced directly from the asymptotic expansion of $\bar{K}\left(s^{2}\right)$ for $s \rightarrow \infty$, where

$$
\bar{K}\left(s^{2}\right)=\int_{\theta=0}^{2 \pi} \int_{r=a}^{b} r \bar{\chi}\left(r, \theta, r, \theta ; s^{2}\right) d r d \theta .
$$

\section{Construction of Green's function}

It is well known that the membrane equation (2.8) has the fundamental solution

$$
\begin{aligned}
\bar{G}_{0}\left(r, \theta, r^{\prime}, \theta^{\prime} ; s^{2}\right) & =\frac{1}{2 \pi} K_{0}\left(s\left|\mathbf{x}-\mathbf{x}^{\prime}\right|\right) \\
& =\frac{1}{2 \pi} \sum_{m=-\infty}^{\infty} I_{m}\left(s r^{\prime}\right) K_{m}(s r) \cos \left[m\left(\theta-\theta^{\prime}\right)\right]
\end{aligned}
$$

where $K_{0}$ is the modified Bessel function of the second kind and of zero order, (see, for example [8]).

On solving the membrane equation (2.8) we deduce that if $r^{\prime} \leqslant r \leqslant b$,

$$
\begin{aligned}
\bar{G} & \left(r, \theta, r^{\prime}, \theta^{\prime} ; s^{2}\right) \\
\quad & \sum_{m=-\infty}^{\infty}\left\{\frac{1}{2 \pi} K_{m}(s r) I_{m}\left(s r^{\prime}\right)+A_{m} K_{m}(s r)+B_{m} I_{m}(s r)\right\} \cos \left[m\left(\theta-\theta^{\prime}\right)\right],
\end{aligned}
$$


and if $r^{\prime} \geqslant r \geqslant a$,

$$
\begin{aligned}
& \bar{G}\left(r, \theta, r^{\prime}, \theta^{\prime} ; s^{2}\right) \\
& \quad=\sum_{m=-\infty}^{\infty}\left\{\frac{1}{2 \pi} K_{m}\left(s r^{\prime}\right) I_{m}(s r)+A_{m} K_{m}(s r)+B_{m} I_{m}(s r)\right\} \cos \left[m\left(\theta-\theta^{\prime}\right)\right],
\end{aligned}
$$

where $A_{m}$ and $B_{m}$ are constants to be determined.

Consequently, it is straightforward to show that at $r^{\prime}=r$ and $\theta^{\prime}=\theta$ the equation (2.8) has the regular solution

$$
\begin{aligned}
\bar{\chi}\left(r, \theta, r, \theta ; s^{2}\right)=\sum_{m=-\infty}^{\infty} \frac{1}{2 \pi R_{m}} & \left\{\left[s I_{m}^{\prime}(s a)+\gamma_{1} I_{m}(s a)\right]\right. \\
& \times\left[s I_{m}^{\prime}(s b)+\gamma_{2} I_{m}(s b)\right] K_{m}^{2}(s r) \\
& -2\left[s I_{m}^{\prime}(s a)+\gamma_{1} I_{m}(s a)\right] \\
& \times\left[s K_{m}^{\prime}(s b)+\gamma_{2} K_{m}(s b)\right] I_{m}(s r) K_{m}(s r) \\
& +\left[s K_{m}^{\prime}(s a)+\gamma_{1} K_{m}(s a)\right] \\
& \left.\times\left[s K_{m}^{\prime}(s b)+\gamma_{2} K_{m}(s b)\right] I_{m}^{2}(s r)\right\},
\end{aligned}
$$

where

$$
\begin{aligned}
R_{m}=\left[s I_{m}^{\prime}(s a)+\gamma_{1} I_{m}(s a)\right]\left[s K_{m}^{\prime}(s b)+\gamma_{2} K_{m}(s b)\right] \\
-\left[s K_{m}^{\prime}(s a)+\gamma_{1} K_{m}(s a)\right]\left[s I_{m}^{\prime}(s b)+\gamma_{2} I_{m}(s b)\right] \neq 0 .
\end{aligned}
$$

If we insert (3.4) into (2.9) and integrate, we find after some reduction that

$$
\bar{K}\left(s^{2}\right)=\frac{a^{2}}{2} \sum_{m=-\infty}^{\infty} f_{1}(m ; s)-\frac{b^{2}}{2} \sum_{m=-\infty}^{\infty} f_{2}(m ; s),
$$

where

$$
\begin{aligned}
f_{1}(m ; s)= & \left(1+\frac{m^{2}}{s^{2} a^{2}}\right)\left\{I_{m}(s a) K_{m}(s a)+\frac{I_{m}(s a)}{a\left[s I_{m}^{\prime}(s a)+\gamma_{1} I_{m}(s a)\right]}\right\} \\
& -I_{m}^{\prime}(s a) K_{m}^{\prime}(s a)+\frac{\gamma_{1} I_{m}^{\prime}(s a)}{s a\left[s I_{m}^{\prime}(s a)+\gamma_{1} I_{m}(s a)\right]} \\
& +\left[\frac{\gamma_{1}^{2}}{s^{2}}-\left(1+\frac{m^{2}}{s^{2} a^{2}}\right)\right] \frac{\left[s I_{m}^{\prime}(s b)+\gamma_{2} I_{m}(s b)\right]}{a^{2} R_{m}\left[s I_{m}^{\prime}(s a)+\gamma_{1} I_{m}(s a)\right]}
\end{aligned}
$$


and

$$
\begin{aligned}
f_{2}(m ; s)= & \left(1+\frac{\dot{m}^{2}}{s^{2} b^{2}}\right)\left\{I_{m}(s b) K_{m}(s b)-\frac{I_{m}(s b)}{b\left[s I_{m}^{\prime}(s b)+\gamma_{2} I_{m}(s b)\right]}\right\} \\
& -I_{m}^{\prime}(s b) K_{m}^{\prime}(s b)-\frac{\gamma_{2} I_{m}^{\prime}(s b)}{s b\left[s I_{m}^{\prime}(s b)+\gamma_{2} I_{m}(s b)\right]} \\
& +\left[\frac{\gamma_{2}^{2}}{s^{2}}-\left(1+\frac{m^{2}}{s^{2} b^{2}}\right)\right] \frac{\left[s I_{m}^{\prime}(s a)+\gamma_{1} I_{m}(s a)\right]}{b^{2} R_{m}\left[s I_{m}^{\prime}(s b)+\gamma_{2} I_{m}(s b)\right]} .
\end{aligned}
$$

As $\gamma_{1}, \gamma_{2} \rightarrow \infty$, we recover (2.1.3) and (2.1.4) of [7]. The series (3.6) is slowly convergent for $s \rightarrow \infty$ and it is therefore expedient to apply a Watson transformation [9] to obtain

$$
\bar{K}\left(s^{2}\right) \sim a^{2} \int_{0}^{\infty} f_{1}(\nu ; s) d \nu-b^{2} \int_{0}^{\infty} f_{2}(\nu ; s) d \nu \text { as } s \rightarrow \infty .
$$

It now follows that the functions $f_{1}(\nu ; s)$ and $f_{2}(\nu ; s)$ may be expressed in terms of the asymptotic expansions of the modified Bessel functions and their derivatives due to Olver [4]; these expansions for $s \rightarrow \infty$ are uniformly valid in $\nu$ for $|\arg \nu|<\pi / 2$.

\section{Construction of $\theta(t)$ for our impedance problem}

In this section, we look at the following cases:

Case $1 .\left(0<\gamma_{1}, \gamma_{2} \ll 1\right)$

In this case we deduce after some reduction that

$$
\begin{aligned}
& f_{1}(\nu ; s) \sim \frac{\left(\nu^{2}+s^{2} a^{2}\right)^{1 / 2}}{s^{2} a^{2}} \sum_{n=0}^{\infty} \frac{A_{1, n}(\tau)}{\nu^{n}} \text { as } s \rightarrow \infty, \\
& f_{2}(\nu ; s) \sim \frac{\left(\nu^{2}+s^{2} b^{2}\right)^{1 / 2}}{s^{2} b^{2}} \sum_{n=0}^{\infty} \frac{A_{2, n}(\eta)}{\nu^{n}} \quad \text { as } s \rightarrow \infty,
\end{aligned}
$$

where $\tau=\nu /\left(\nu^{2}+s^{2} a^{2}\right)^{1 / 2}, \eta=\nu /\left(\nu^{2}+s^{2} b^{2}\right)^{1 / 2}$ and for $n=0,1,2,3$

$$
\begin{aligned}
& A_{1,0}=0, \quad A_{1,1}=\frac{1}{2}\left(\tau-\tau^{3}\right), \quad A_{1,2}=\tau^{2}\left(\gamma_{1} a-\frac{1}{2}\right)-\tau^{4}\left(\gamma_{1} a-\frac{3}{2}\right)-\tau^{6}, \\
& A_{1,3}=\tau^{3}\left(\frac{3}{8}-\gamma_{1} a+\gamma_{1}^{2} a^{2}\right)+\tau^{5}\left(-\frac{23}{8}+3 \gamma_{1} a-\gamma_{1}^{2} a^{2}\right)+\tau^{7}\left(\frac{41}{8}-2 \gamma_{1} a\right)-\frac{21}{8} \tau^{9} \text {, } \\
& A_{2,0}=0, \quad A_{2,1}=-\frac{1}{2}\left(\eta-\eta^{3}\right), \quad A_{2,2}=\eta^{2}\left(\gamma_{2} b-\frac{1}{2}\right)-\eta^{4}\left(\gamma_{2} b-\frac{3}{2}\right)-\eta^{6}, \\
& A_{2,3}=-\eta^{3}\left(\frac{3}{8}-\gamma_{2} b+\gamma_{2}^{2} b^{2}\right)-\eta^{5}\left(-\frac{23}{8}+3 \gamma_{2} b-\gamma_{2}^{2} b^{2}\right)-\eta^{7}\left(\frac{41}{8}-2 \gamma_{2} b\right)+\frac{21}{8} \eta^{9} \text {. }
\end{aligned}
$$


If the asymptotic expansions (4.1) and (4.2) are now integrated, we deduce that:

$$
\begin{aligned}
& \bar{K}\left(s^{2}\right) \sim \frac{\pi(a+b)}{4 s}-\frac{\left(\gamma_{2} b-\gamma_{1} a\right)}{s^{2}} \\
&+\left\{7\left(\frac{1}{a}+\frac{1}{b}\right)-32\left(\gamma_{1}+\gamma_{2}\right)+64\left(\gamma_{1}^{2} a+\gamma_{2}^{2} b\right)\right\} \frac{\pi}{256 s^{3}}+O\left(\frac{1}{s^{4}}\right) \\
& \text { as } s \rightarrow \infty .
\end{aligned}
$$

On inverting Laplace transforms and using (2.5) we have the spectral formula:

$$
\begin{aligned}
\theta(t) \sim & \frac{b^{2}-a^{2}}{4 t}+\frac{\pi^{1 / 2}(a+b)}{4 t^{1 / 2}}-\left(\gamma_{2} b-\gamma_{1} a\right) \\
+ & \left\{7\left(\frac{1}{a}+\frac{1}{b}\right)-32\left(\gamma_{1}+\gamma_{2}\right)+64\left(\gamma_{1}^{2} a+\gamma_{2}^{2} b\right)\right\} \frac{(\pi t)^{1 / 2}}{128}+O(t) \\
\text { as } t & \rightarrow 0 .
\end{aligned}
$$

Similarly the following asymptotic spectral formulae may be derived:

Case 2. $\left(0<\gamma_{1} \ll 1, \gamma_{2} \gg 1\right)$

$$
\begin{aligned}
& \theta(t) \sim \frac{b^{2}-a^{2}}{4 t}+\frac{\pi^{1 / 2}\left[a-\left(b+\gamma_{2}^{-1}\right)\right]}{4 t^{1 / 2}}-\gamma_{1} a \\
&+\left\{\frac{7}{a}+\frac{1}{b}-32 \gamma_{1}+64 \gamma_{1}^{2} a-\frac{\gamma_{2}^{-1}}{b^{2}}\right\} \frac{(\pi t)^{1 / 2}}{128}+O(t) \\
& \text { as } t \rightarrow 0 .
\end{aligned}
$$

Case 3. $\left(\gamma_{1} \gg 1,0<\gamma_{2} \ll 1\right)$

$$
\begin{aligned}
& \theta(t) \frac{b^{2}-a^{2}}{4 t}+\frac{\pi^{1 / 2}\left[b-\left(a+\gamma_{1}^{-1}\right)\right]}{4 t^{1 / 2}}-\gamma_{2} b \\
&+\left\{\frac{1}{a}+\frac{7}{b}-32 \gamma_{2}+64 \gamma_{2}^{2} b-\frac{\gamma_{1}^{-1}}{a^{2}}\right\} \frac{(\pi t)^{1 / 2}}{128}+O(t) \\
& \text { as } t \rightarrow 0 .
\end{aligned}
$$

This derives from Case 2 with the interchanges $a \leftrightarrow b$ and $\gamma_{1} \leftrightarrow \gamma_{2}$ in the terms other than the first. 
Case 4. $\left(\gamma_{1}, \gamma_{2} \gg 1\right)$

$$
\begin{gathered}
\theta(t) \sim \frac{b^{2}-a^{2}}{4 t}-\frac{\pi^{1 / 2}\left[\left(a+\gamma_{1}^{-1}\right)+\left(b+\gamma_{2}^{-1}\right)\right]}{4 t^{1 / 2}} \\
+\left\{\frac{1}{a}+\frac{1}{b}-\frac{\gamma_{1}^{-1}}{a^{2}}-\frac{\gamma_{2}^{-1}}{b^{2}}\right\} \frac{(\pi t)^{1 / 2}}{128}+O(t) \\
\text { as } t \rightarrow 0 .
\end{gathered}
$$

We remark that

(4.4) agrees with (1.5) if $\gamma_{1}=\gamma_{2}=0$;

(4.5) agrees with (1.6) if $\gamma_{1}=0$ and $\gamma_{2} \rightarrow \infty$;

(4.6) agrees with (1.7) if $\gamma_{1} \rightarrow \infty$ and $\gamma_{2}=0$;

(4.7) agrees with (1.8) if $\gamma_{1}=\gamma_{2} \rightarrow \infty$.

The asymptotic expansions (4.4)-(4.7) may be interpreted as:

(i) $D$ is a circular annulus and we have the impedance boundary conditions of (1.4) on both boundaries of $D$ with large/small impedances $\gamma_{1}, \gamma_{2}$ as indicated in the specifications of the four respective cases.

(ii) For the first three terms, $D$ is a bounded domain of area $\pi\left(b^{2}-a^{2}\right)$.

In Case 1 , it has $h=\left[1+6\left(\gamma_{2} b-\gamma_{1} a\right)\right]$ holes, a boundary of length $2 \pi(a+b)$ together with Neumann conditions on the boundaries, provided $h$ is an integer.

In Case 2, it has $h=\left(1+6 \gamma_{1} a\right)$ holes, a part of the boundary of length $2 \pi a$ with Neumann conditions and the other part of length $2 \pi\left(b+\gamma_{2}^{-1}\right)$ together with Dirichlet conditions, provided $h$ is an integer.

In Case 4, it has only one hole $(h=1)$, a boundary of length $2 \pi\left\{\left(a+\gamma_{1}^{-1}\right)+\right.$ $\left.\left(b+\gamma_{2}^{-1}\right)\right\}$ together with Dirichlet conditions on the boundaries.

(iii) The fourth and further terms in (4.4)-(4.7), as yet undetermined, would require different interpretations.

(iv) If it is known that the domain $D$ is a circular annulus, then both the coefficients of $t^{-1 / 2}$ and that of $t^{1 / 2}$ in (4.7) may be solved to determine $\gamma_{1}$ and $\gamma_{2}$.

(v) If, in the formula (4.4), $\gamma_{1} / \gamma_{2}=b / a$ then the first three terms agree with the annulus with Neumann conditions. Also, if further $\gamma_{1} a=\frac{1}{2}=\gamma_{2} b$, then the first four terms agree with the annulus with Neumann conditions (i.e. with the case obtained by setting $\gamma_{1}=\gamma_{2}=0$ in (4.4)).

\section{Acknowledgements}

The author would like to express his sincere thanks to the referees for their interesting suggestions and comments. He also would like to thank the editors, Professor E. O. Tuck and Dr. C. E. M. Pearce for their suggestions. 


\section{References}

[1] H. P. W. Gottlieb, "Hearing the shape of an annular drum", J. Austral. Math. Soc. Ser. B 24 (1983) 435-438.

[2] H. P. W. Gottlieb, "Eigenvalues of the Laplacian with Neumann boundary conditions", $J$. Austral. Math. Soc. Ser. B 26 (1985) 293-309.

[3] M. Kac, "Can one hear the shape of a drum?", Amer. Math. Monthly 73, No. 4, Part 11 (1966) $1-23$.

[4] F. W. J. Olver, "The asymptotic expansion of Bessel functions of large order", Philos. Trans. Roy. Soc. London Ser. A 247 (1954) 328-368.

[5] A. Pleijel, "A study of certain Green's functions with applications in the theory of vibrating membranes", Ark. Mat. 2 (1953) 553-569.

[6] B. D. Sleeman and E. M. E. Zayed, "An inverse eigenvalue problem for a general convex domain", J. Math. Anal. Appl. 94 No. 1 (1983) 78-95.

[7] B. D. Sleeman and E. M. E. Zayed, "Trace formulae for the eigenvalues of the Laplacian," $Z$. Angew. Math. Phys. 35 (1984) 106-115.

[8] I. Stakgold, Green's functions and boundary value problems (John Wiley Sons, New York, 1979).

[9] K. Stewartson and R. T. Waechter, "On hearing the shape of a drum: further results", Proc. Camb. Phil. Soc. 69 (1971) 353-363. 